\title{
Quantum properties of a parametric four-wave mixing in a Raman type atomic system
}

\author{
A.V. Sharypov ${ }^{1,2, *}$, Bing $\mathrm{He}^{3}$, V.G. Arkhipkin ${ }^{1}$, and S.A. Mislivets ${ }^{1}$ \\ ${ }^{1}$ Kirensky Institute of Physics, Federal Research Center KSC SB RAS, 50 Akademgorodok, \\ Krasnoyarsk 660036, Russia \\ ${ }^{2}$ Science Center "Newton Park", 1 Mira Str., Krasnoyarsk, 660049 Russia \\ ${ }^{3}$ Department of Physics, University of Arkansas, Fayetteville, AR 72701, USA
}

\begin{abstract}
We present a study of the quantum properties of two light fields used to parametric four-wave mixing in a Raman type atomic system. The system realizes an effective Hamiltonian of beamsplitter type coupling between the light fields, which allows to control squeezing and amplitude distribution of the light fields, as well as realizing their entanglement. The scheme can be feasibly applied to engineer the quantum properties of two single-mode light fields in properly chosen input states.
\end{abstract}

Let us consider a three-level atomic system with the ground state $\mid a>$ and two upper states $\mid \mathrm{b}>$ and $\mid \mathrm{c}>$; see Fig. 1 .

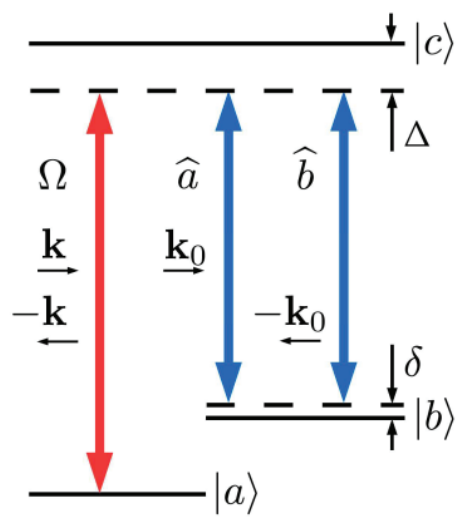

Fig. 1. Scheme of a Raman-type dispersive four-wave mixing.

Through the system the input quantum light fields of single mode, the blue ones in Fig. 1, effectively interact with one another. The classical standing electromagnetic field with the Rabi frequency $\Omega$ implements the transition $|\mathrm{a}\rangle-|\mathrm{c}\rangle$. Such classical standing wave is applied along $\mathrm{z}$-direction and can be decomposed into two running wave with the wave vectors $\pm \mathrm{k}$.

\footnotetext{
*Corresponding author: asharypov@yandex.ru
} 
The two counterpropagating quantum modes that are coupled to the transition $|\mathrm{b}\rangle-\mid \mathrm{c}>$ with the coupling parameter $g$ also propagate along $\mathrm{z}$-direction.

In order to avoid one- and two-photon transitions we use large one ( $\triangle$ )- and two-photon $(\delta)$ detuning

$$
\begin{gathered}
|\Omega / \Delta|<<1, \\
|\Omega g(n) 1 / 2 /(\Delta \delta)|<<1,
\end{gathered}
$$

where $\mathrm{g}-$ is the coupling strenght.

The derived effective Hamiltonian for the present system has the similar form to a beamsplitter Hamiltonian. Further we demonstrate that there are possibilities

for the parametric amplitude amplification (Fig.2) of a squeezed state, as well as the squeezing transferring (Fig.3), with such type of interaction.

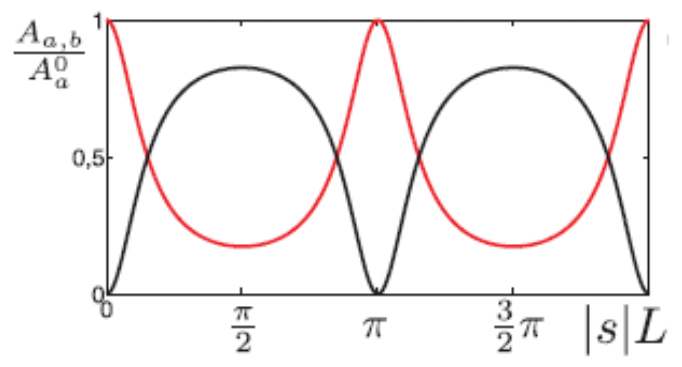

Fig. 2. Dynamics of the normalized quantum field's amplitude as a function of the dimensionless medium length.

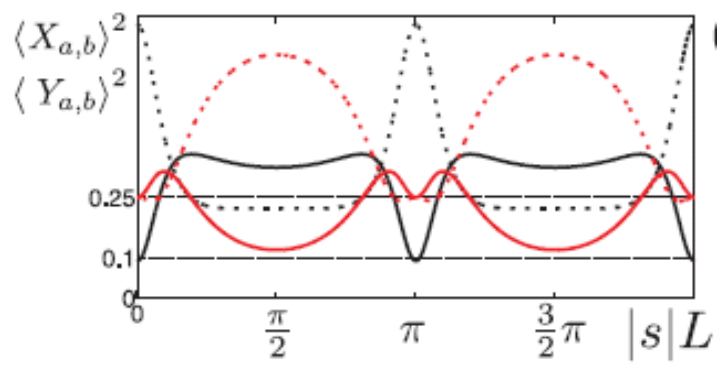

Fig. 3. Dynamics of the fields quadratures fluctuations as a function of the dimentionless medium length.

This work was supported by the Russian Foundation for Basic Research under Grant No 15-02-03959. 